\title{
Adolescent psychopathology and psychological wellbeing: a network analysis approach
}

\author{
Stephanie Campbell ${ }^{* \dagger}$ and Tom L. Osborn ${ }^{\dagger}$
}

\begin{abstract}
Background: The extent to which psychological wellbeing may play a preventive and therapeutic role in the development and maintenance of adolescent emotional disorders depends, in part, on the nature of the overlap between these two constructs. We estimated network analysis to examine the relationship between adolescent psychopathology (measured by depression and anxiety symptoms) and psychological wellbeing (measured by happiness, optimism, social support, perceived control, and gratitude).
\end{abstract}

Methods: This was a cross-sectional study with a large community sample of Kenyan adolescents ( $N=2192$, aged 13-18). Network analyses were conducted to examine the topology, stability, centrality, and bridge nodes of a network of psychopathology and psychological wellbeing measures.

Results: Two distinct community clusters emerged, one for psychopathology nodes and another for wellbeing nodes, suggesting that these are two distinct but connected concepts. Central and bridge nodes of the wellbeing and psychopathology network were identified. The most central nodes in the network were family provides emotional help and support and self-blame; the strongest negative edges between psychopathology and psychological wellbeing were depressed mood — l love life and irritability—l am a joyful person; the main bridge nodes were family helps me and I can talk to family about problems.

Conclusions: Our findings expand understanding of the relationship between psychopathology and wellbeing in an understudied population and are suggestive of how psychological wellbeing can inform psychopathological treatment and preventive efforts in low-income regions such as those in Sub Saharan Africa.

Keywords: Adolescents, Africa, Network analysis, Psychopathology, Mental health, Depression, Anxiety, Well-being, Happiness, Social support

\section{Background}

Adolescent mood and anxiety disorders affect a significant portion of the global adolescent population, accounting for $45 \%$ of the global disease burden on youths aged 15-19 [1]. Even below a clinical threshold, depression and anxiety are associated with many negative physical, social, behavioral, and health outcomes, which can

\footnotetext{
* Correspondence: campbell@shamiri.institute

${ }^{\dagger}$ These authors contributed equally to this work and share first authorship. Shamiri Institute, Nairobi, Kenya
}

last a lifetime [2,3], such as increased risks in health, behavior, and education [4]. Nearly half of all behavioral and emotional disorders have an initial onset during adolescence [5], increasing the risk of these disorders in adulthood by 2-to-3 times [6]. Therefore, this transitional phase of adolescence is a critical time to prevent and treat emotional disorders like depression and anxiety.

One approach to combat youth mental health disorders might lie in the promotion of positive aspects of

(c) The Author(s). 2021 Open Access This article is licensed under a Creative Commons Attribution 4.0 International License, which permits use, sharing, adaptation, distribution and reproduction in any medium or format, as long as you give appropriate credit to the original author(s) and the source, provide a link to the Creative Commons licence, and indicate if changes were made. The images or other third party material in this article are included in the article's Creative Commons licence, unless indicated otherwise in a credit line to the material. If material is not included in the article's Creative Commons licence and your intended use is not permitted by statutory regulation or exceeds the permitted use, you will need to obtain permission directly from the copyright holder. To view a copy of this licence, visit http://creativecommons.org/licenses/by/4.0/ The Creative Commons Public Domain Dedication waiver (http://creativecommons.org/publicdomain/zero/1.0/) applies to the data made available in this article, unless otherwise stated in a credit line to the data. 
psychological functioning, such as psychological wellbeing, which may play a preventative and therapeutic role in the development of emotional disorders [7, 8]. Broadly defined, psychological wellbeing refers to an individual's condition of experiencing their lives positively [9]. It can include the positive feelings of satisfaction, accomplishment, and fulfillment in diverse life domains as well as the lack of negative aspects like mental illness and loneliness [10]. Given its multiplicity, psychological wellbeing is measured by many models, including PERMA (Positive Emotion, Engagement, Relationships, Meaning and Accomplishment; [11]), subjective wellbeing (SWB; [12]), and community quality of life (QoL; [13]). Gratitude, social support, self-perception, and happiness have also been used to measure psychological wellbeing [14, 15].

The extent to which psychological wellbeing plays a preventative or therapeutic role in adolescent psychopathology depends partly on the nature of the overlap between the two [7]. Many studies have shown that psychological wellbeing and psychopathology are strongly and negatively associated [16, 17]. Indeed, the strength of this association-generally, correlations of $r=-0.40$ to $r=-0.55$-suggests that these two constructs may be two distinct but overlapping dimensions of functioning [7]. Observers have described this relationship through a binary continuum model, as well as through a dualfactor model. In the binary continuum model, psychological wellbeing and psychopathology are on the extreme opposite ends of a wellbeing band [18]. In the dual-factor model, the two constructs are distinct but related $[8,19]$. One study assessed Australian adolescents $(N=345)$ for psychological wellbeing (measured by happiness, life satisfaction, and positive affect scales) and psychopathology (measured by depression, anxiety, and negative affect scales), which revealed two factors for psychological wellbeing and youth psychopathology, respectively [20]. Another study used the dual-factor model along with self-report scores to classify Canadian youths $(N=407)$ into four categories: (1) high psychological wellbeing and low psychopathy, (2) low psychological wellbeing and high psychopathy, (3) low psychological wellbeing and low psychopathy, and (4) high psychological wellbeing and high psychopathy [8]. Another study with American adolescents $(N=349)$ found that by using the cutoff norms for the Child Behavior Checklist and corresponding psychological wellbeing scores, adolescents could also be classified into one of the four groups mentioned above, providing further evidence that psychological wellbeing and adolescent psychopathology may be distinct but interrelated [19].

Beyond the evidence from many cross-sectional studies, one recent Dutch study investigated the extent to which the association between psychological wellbeing and psychopathology is a function of correlated genetic and/or correlated environmental factors [7]. In the study, psychological wellbeing was assessed (measured by subjective happiness, quality of life, and satisfaction with life) for a large population-based cohort of adolescent twins and their non-twin siblings $(N=9136$ and 1474, respectively). Psychopathology was also assessed (measured by all syndrome and broad band based scales of the Achenbach System of Empirically Based Assessments (ASEBA) Youth Self Report Scale). The study found significant negative associations between psychological wellbeing and psychopathology, which were primarily explained by genetic correlations [7]. This finding--that a genetic liability to lower levels of psychological wellbeing may be suggestive of genetic liability to higher levels of psychopathology--supports the use of psychological wellbeing measures to screen for adolescent psychopathology before the presence of clear signs of psychopathology [7].

The use of psychological wellbeing indices to combat adolescent psychopathology may be of particular importance for adolescent populations in Sub Saharan Africa (SSA). Not only are there high prevalence rates of adolescent depression and anxiety symptoms in SSA countries [21], there is currently a dearth of research with this population, limiting the knowledge of rates, comorbidity, correlates, predictors, protective factors, and treatment options in this region [22]. In SSA, treatment options for psychopathology are limited [2, 21], government spending on mental healthcare is minimal [21], and societal stigma against mental illnesses dissuades many from seeking help [23]. Indeed, research that espouses the association between psychological wellbeing and adolescent psychopathology with SSA populations may be of public policy utility in this region. For example, the societal stigma around mental health limits help-seeking amongst Kenyan adolescents, many of whom do not want to be diagnosed with mental disorders, much less seek treatment for it [23]. If we could use psychological wellbeing indices (like gratitude and happiness) to inform the public health efforts on the screening, prevention, and treatments for youth mental disorders, then these efforts could be done in a potentially non-stigmatizing manner.

One way of expanding our knowledge of the relationship between psychological wellbeing and adolescent psychopathology might lie in the use of network analysis-a novel conceptual model in which a psychological construct is conceptualized as the interplay of traits or symptoms that influence each other [24]. In a network structure, a psychological construct (e.g., a symptom or a trait) is represented by a node, and the relationship between each pair of constructs is depicted by an edge 
between the corresponding nodes. Networks allow for the identification of the central symptoms or traits of a psychological construct, which are likely to activate the entire network and might be sites for direct targeting in prevention and treatment [25]. The network framework has been used to circumvent some of the theoretical and psychometric limitations of traditional models [26], such as the classification of the symptoms into discrete mental disorders by the DSM [27]. Classification models are problematic since mental disorders share a broad spectrum of overlapping symptoms [24] and since specific disorders may have a wide range of symptoms with different treatments [28]. As network analysis conceptualizes psychiatric disorders as systems the emerge from symptoms interactions and not an underlying disease entity, they embrace and account for the comorbidity and heterogeneity of emotional disorders [29].

In addition to identifying and targeting the central symptoms of an emotional disorder for reduction and the central traits of psychological wellbeing for enhancement, network analysis can help shed light on the connectivity between psychological wellbeing and adolescent psychopathology. Specifically, we can identify an individual psychological wellbeing trait that is highly connected to a particular emotional disorder symptom and quantify the nature of that connectivity. Overall, network analysis can allow us to study the structure of psychopathology and psychological wellbeing, jointly.

In the present study, we used network analysis to analyze the structure of psychological wellbeing indicators and symptoms of depression and anxiety in a large community sample of adolescents in Kenya. Happiness, gratitude, optimism, perceived control, and social support measures were used to assess psychological wellbeing, while depression and anxiety symptoms were used to assess adolescent psychopathology. The aims of our study were to investigate: (1) the structures and clusters that the indicators of psychological wellbeing and the symptoms of depression and anxiety form in a network--i.e., whether psychological wellbeing items form a distinct cluster or overlap with the symptoms of psychopathology, (2) the central nodes (i.e., symptoms or traits) in a network of psychological wellbeing and youth psychopathology, and (3) the important "bridge" nodes that connect the community cluster of psychological wellbeing and the community cluster of adolescent psychopathology. The present study is, to the best of our knowledge, the first of its kind to investigate psychological wellbeing and youth depression and anxiety symptoms in a community sample of SSA adolescents.

\section{Methods}

\section{Participants and procedures}

Participants were 2192 Kenyan adolescents recruited from four secondary schools in Nairobi and Kiambu
Counties, as part of a large-scale clinical trial (called Shamiri [30, 31];). The trial was registered in the Pan African Clinical Trials Registry (PACTR201906525818462). Participants provided self-report data on depression, anxiety, psychological wellbeing measures, and other health-related and socio-demographic variables by completing a baseline questionnaire battery in their classrooms. There was a slight female majority $(N=1246$; $58.3 \%)$, and the mean age was $15.21(\mathrm{SD}=1.14)$. See Table 1 for full demographic information and descriptive statistics. Using a clinical cutoff of 10-per clinical guidelines from North American samples [34, 35] that have been used previously with Kenyan youths [36, 37], some $28.56 \%$ of participants $(N=626)$ met the criteria for moderate-to-severe depression and $26.55 \%(N=582)$ met that of moderate-to-severe anxiety. All study procedures were approved by the Maseno University Ethics Review Committee (MUERC, No. MUERC/727/19) before the start of data collection. All adolescents were eligible to participate if they consented and were enrolled in the participating schools. Parental consent and written informed consent and assent were obtained for all adolescents per research ethics procedures at MUERC. Data used for the present study is stored in the Open Science Framework repository and is publicly available (DOI: https://doi.org/10.17605/OSF.IO/8M5D9).

\section{Measures}

\section{Adolescent psychopathology measures}

Depressive symptoms were assessed using the 8-item version of the Patient Health Questionnaire (PHQ-9; $[34,38])$, a self-report questionnaire used to assess the severity of depressive symptoms. PHQ-8 and PHQ-9 scores are highly correlated, and the same cutoffs can be used to assess depression severity [38]. A previous study has documented that the PHQ-8 demonstrated adequate psychometric properties for the PHQ-8 with Kenyan adolescents [32, 39] and adults [40, 41]. Anxiety symptoms were assessed using a 7-item self-report questionnaire, the Generalized Anxiety Disorder Screener (GAD-7; [35]). Like with the PHQ-8, a previous has documented

Table 1 Participant demographic information

\begin{tabular}{lllr}
\hline Characteristic & N (\%) & M (SD) & Range \\
\hline Age & & $15.21(1.14)$ & $14-20$ \\
Gender & & & \\
$\quad$ Female & $1246(56.80)$ & & \\
$\quad$ Male & $890(40.60)$ & & \\
School type & & \\
$\quad$ National & $1715(78.2)$ & \\
$\quad$ Rural (sub-county) & $476(21.7)$ & \\
\hline
\end{tabular}

More information regarding school type classification in Kenya is available elsewhere [32, 33] 
adequate psychometric properties with Kenyan youths [32].

\section{Psychological wellbeing measures}

Happiness and optimism were assessed using two subscales of the EPOCH Measure of Adolescent of WellBeing (EPOCH; [42]): optimism and happiness. Social support was self-reported using the Family, Friends, and Significant Other sub-scales of the Multidimensional Scale of Perceived Social Support (MSSS; [43]). Perceived academic control measures adolescent perception of their competency and regulation over that competency and was assessed using the academic sub-scale of the Perceived Control Scale (PSC; [44]). Gratitude was measured using the 6-item Gratitude Questionnaire (GQ-6; [45]). Gratitude is strongly related to well-being and mental health, a link that has been suggested to be unique and causal [46]. We also collected sociodemographic information such as age, gender, form, tribe, county, economic status, parental education, and family members.

\section{Statistical analysis}

We performed all our analysis in $R$. First, we investigated the presence of redundant nodes, or overlapping symptoms, by checking pairs of nodes for high correlation $(\mathrm{r}>.5)$ and similar correlation patterns with all the other nodes via the networktools package in $R$ [47]. Second, we estimated the network models for the data and validated their accuracy and stability using the bootnet package in $R$ [48]. Third, we plotted the network structures and computed the centrality measures using the qgraph package in $R$ [49]. Fourth and lastly, we investigated bridge symptoms, the main symptoms that connect clusters of symptoms, with the networktools package in $R$ [47].

To deal with missingness, we conducted multiple imputations using Fully Conditional Specification (FCS) for missing item-level data that was implemented using the multivariate imputation by chain equation (mice) algorithm in $R[50,51]$ under the assumption that data were missing at random. Specifically, we used the predictive mean matching approach to impute missing-item level data fifty times; estimates were then pooled to get one overall set of parameter estimates. The $R$ code for our statistical analysis is publicly available (DOI: https://doi. org/10.17605/OSF.IO/8M5D9).

\section{Node selection}

The lack of variance in variables may lead to the misinterpretation of network structure. If two nodes represent the same construct, there will be redundancy in the network, which can be problematic for interpretability [25]. Thus, redundant pairs should not be included together in the network. In contrast, if two nodes represent independent constructs, we would expect their correlation patterns with other nodes to vary. We tested for the problematic presence of multiple symptoms representing the same underlying construct using the goldbricker procedure [47], which checks each pair of nodes for two indicators of redundancy: a high correlation between them $(r>.5)$ and a proportion of significantly different correlations with all other nodes under a certain threshold (we used a $20 \%$ threshold).

\section{Network estimation and accuracy}

In psychopathology, a network consists of symptoms and the psychometric associations between them. These associations are not explicitly present in a dataset, but they can be estimated by computing partial correlations between the symptoms, controlling for all other symptom connections [48]. We used partial.r, which finds the residuals of various correlations and then correlates these residuals to partial the effect of variables like gender, age, and school type. Next, we estimated a Gaussian graphical model (GGM) to estimate regularized partial correlation networks for psychological wellbeing symptoms and psychiatric symptoms using the bootnet package in $R[29,48]$. We regularized the GGM with the graphical Least Absolute Shrinkage and Selection Operator (LASSO) method to find the best-fitting by penalizing, or shrinking, small edge values estimated in the network. LASSO also helps address the multiple testing problem by controlling false-positive errors. (LASSO regularization techniques are commonly performed in this type of network analysis $[52,53]$.) The best-fitting model was found with the EBICglasso procedure, which selects the optimal degree of shrinkage according to an Extended Bayesian Information Criterion (EBIC) and hyperparameter set to 0.5 [54]. To plot the networks, we used the qgraph package in $R$.

\section{Node centrality}

A highly central node is a node that has particular structural importance in the network based on the strength of its connection to other nodes. The centrality of a node can be used to infer its influence, or structural importance, in the network. There are many indices used to estimate centrality: betweenness-how a node influences the average path between other pairs of nodes, closeness-how a node is indirectly connected to the other nodes, strength-how a node is directly connected to the other nodes, and expected influence (EI) - how a node is connected to the sum of all edge weights [55]. As partial correlations were used to estimate the networks, it was not fitting to use the closeness or betweenness indices. Instead, we relied on the strength index to estimate the symptoms that should be most directly 
targeted in treatment. The qgraph package in $R$ was used [48].

\section{Network accuracy and stability}

Bootstrapping was used to determine the accuracy and stability of the networks [48]. First, we tested the accuracy of each network using nonparametric bootstrapping, a process that repeatedly resamples subsets of the data to calculate a confidence interval (CI) as the range of bootstrapped values from different sampling levels. We first used 15,000 bootstraps--or 15,000 repetitions of estimating the model with sampled data and calculating the $95 \%$ confidence intervals (CIs)--to assess the accuracy of edge-weights. A large CI indicates that it may be difficult to interpret the edge weight, while a small CI can be interpreted as a precise estimation. Additionally, we enriched the assessment of node importance within the network by using the predictability index, which measures the proportion of variance of each node explained by the neighboring nodes in the network (see [56].) Though similar effects can be achieved with our using of bootstrapping, we estimated the predictability index of our analysis using the $m g m$ package in $R$ (see Additional file 1: Appendix C in Supplementary Materials for further description and results.)

Next, we tested the stability of the centrality indices with case-dropping bootstrapping [48], which is the process of repeatedly estimating a model while dropping rows of the data (i.e., we measure stability while only observing subsets of the data.) We calculated a correlationstability (CS) coefficient, which indicates the maximum proportion of the data that can be dropped while continuing to estimate centrality values that correlate highly $(r>7)$ with the network from the full sample. Scores .25 and .5 indicate benchmarks for adequate and good network stability, respectively [48]. For each network, we created plots displaying the CIs of edges and centrality values, as well as their confidence intervals. Bootstrapped difference plots are useful for estimating which edges or centrality values can be meaningfully interpreted as distinct; we used these plots to guide our interpretations of edge and centrality values.

\section{Bridge nodes}

In a network with multiple scales, bridge nodes are the main nodes that connect to other node clusters, in this case--the other psychiatric and wellbeing measures. We can find these bridge nodes by calculating the bridge centrality statistics from the networktools package in $R$ [47]. The bridge centrality statistic applies to a node's connection to all the other nodes in the other communities to which it does not belong. Bridge strength is defined as the sum of the absolute value of all edges that exist between a given node and all nodes that are not in the same community. Bridge expected influence (onestep) is defined as the sum of the value (either positive or negative) of all edges that exist between a node and all nodes that are in a different community than the node $[47,55]$. Thus, bridge expected influence accounts for how positive and negative edges can neutralize each other. For example, if fasting is positively linked to desire for thinness $(r=.6)$ and negatively to binge eating ( $r=$ -.3 ), then the regular strength centrality for fasting will be 0.9 (sum of absolute values of those edges). However, the bridge expected influence for fasting would be 0.3 because the value of the negative edge is subtracted from the value of the positive edge. Thus, high bridge expected value would indicate that the node is strongly and positively connected to other nodes.

To find bridge nodes, we defined two community clusters in our network. The first community, adolescent psychopathology, included the depression and anxiety symptoms, and the second community, psychological wellbeing, included the gratitude, happiness, optimism, social support, and perceived control items.

\section{Results}

\section{Descriptive statistics and prevalence}

The mean, standard deviation, minimum, maximum, skewness, and kurtosis for both depression and anxiety symptoms are reported in Table 2. The overall mean of the total scores for PHQ and GAD were below the moderate-to-severe cutoff of 10 (PHQ = 7.96; GAD = 7.46). Some $28.56 \%$ of participants endorsed symptoms of moderate-to-severe depression. A higher $26.55 \%$ endorsed symptoms of moderate-to-severe anxiety. For depression, the symptoms with the highest mean ratings were PHQ6 (self-blame) and PHQ7 (trouble concentrating), while those for anxiety were GAD3 (too much worry) and GAD2 (uncontrollable worry). The descriptive statistics for the EPOCH, MSSS, PCS, and GQ-6 items are also reported in Table 2.

\section{Network analysis: adolescent psychopathology and psychological wellbeing Node selection}

Conducting the goldbricker procedure, we found nine pairs of symptoms with less than $20 \%$ significantly different correlations (see Table 3). We first removed the symptoms that were redundant with at least two other symptoms. In other words, we removed MSSS6 (friends try to help me), MSSS5 (I have a special person who is a real source of comfort to me), and PCS5 (I cannot get good grades no matter how hard I try), which allows us to keep MSSS7, MSSS2, MSSS9, MSSS10, PCS4, and PCS7 in the network unaltered. The remaining overlapping symptom pairs were GAD2-3 (uncontrollable worry--too much worry), PCS1-2 (I can get good grades 
Table 2 Mean, standard deviation, minimum, maximum, skewness, and kurtosis of PHQ and GAD symptoms and EPOCH, MSSS, GQ6 and PCS items

\begin{tabular}{|c|c|c|c|c|c|c|c|c|c|c|c|c|c|}
\hline Variable & $M$ & SD & Min & Max & Skewness & Kurtosis & Variable & $M$ & SD & Min & Max & Skewness & Kurtosis \\
\hline $\begin{array}{l}\text { PHQ1: Little interest/ } \\
\text { pleasure }\end{array}$ & 1.13 & 1.00 & 0 & 3 & 0.67 & -0.56 & MSSS3: Family helps me & 6.14 & 1.31 & 1 & 7 & -2.10 & 4.52 \\
\hline $\begin{array}{l}\text { PHQ2: Depressed } \\
\text { mood }\end{array}$ & 0.95 & 0.96 & 0 & 3 & 0.86 & -0.18 & $\begin{array}{l}\text { MSSS4: Family provides emotional } \\
\text { help and support }\end{array}$ & 5.43 & 1.69 & 1 & 7 & -1.15 & 0.48 \\
\hline $\begin{array}{l}\text { PHQ3: Sleep } \\
\text { problems }\end{array}$ & 1.00 & 1.08 & 0 & 3 & 0.76 & -0.73 & $\begin{array}{l}\text { MSSS5: I have a special person is a } \\
\text { real source of comfort }\end{array}$ & 5.23 & 1.80 & 1 & 7 & -0.92 & -0.17 \\
\hline PHQ4: Little energy & 0.89 & 0.92 & 0 & 3 & 0.89 & -0.02 & MSSS6: Friends try to help me & 4.70 & 1.61 & 1 & 7 & -0.68 & -0.14 \\
\hline $\begin{array}{l}\text { PHQ5: Appetite } \\
\text { problems }\end{array}$ & 0.83 & 1.04 & 0 & 3 & 1.02 & -0.26 & MSSS7: I can count on friends & 4.35 & 1.75 & 1 & 7 & -0.44 & -0.75 \\
\hline PHQ6: Self-blame & 1.25 & 1.13 & 0 & 3 & 0.42 & -1.22 & $\begin{array}{l}\text { MSSS8: I can talk to family about } \\
\text { problems }\end{array}$ & 4.77 & 1.92 & 1 & 7 & -0.61 & -0.79 \\
\hline $\begin{array}{l}\text { PHQ7: Trouble } \\
\text { concentrating }\end{array}$ & 1.20 & 1.08 & 0 & 3 & 0.50 & -1.00 & $\begin{array}{l}\text { MSSS9: I have friends to share joys/ } \\
\text { sorrows }\end{array}$ & 4.88 & 1.71 & 1 & 7 & -0.83 & -0.13 \\
\hline $\begin{array}{l}\text { PHQ8: Psychomotor } \\
\text { problems }\end{array}$ & 0.71 & 0.99 & 0 & 3 & 1.24 & 0.32 & $\begin{array}{l}\text { MSSS10: I have a special person } \\
\text { who cares about my feelings }\end{array}$ & 5.41 & 1.77 & 1 & 7 & -1.12 & 0.33 \\
\hline GAD1: Nervousness & 1.10 & 1.01 & 0 & 3 & 0.63 & -0.66 & $\begin{array}{l}\text { MSSS11: Family willing to help me } \\
\text { make decisions }\end{array}$ & 5.76 & 1.52 & 1 & 7 & -1.56 & 1.97 \\
\hline $\begin{array}{l}\text { GAD2: } \\
\text { Uncontrollable worry }\end{array}$ & 1.26 & 1.20 & 0 & 3 & 0.42 & -1.12 & $\begin{array}{l}\text { MSSS12: I can talk about my } \\
\text { problems with friends }\end{array}$ & 4.30 & 1.77 & 1 & 7 & -0.40 & -0.79 \\
\hline $\begin{array}{l}\text { GAD3: Too much } \\
\text { worry }\end{array}$ & 1.31 & 1.26 & 0 & 3 & 0.38 & -1.18 & GQ1: I have a lot to be thankful for & 6.39 & 1.06 & 1 & 7 & -2.79 & 9.24 \\
\hline $\begin{array}{l}\text { GAD4: Trouble } \\
\text { relaxing }\end{array}$ & 0.89 & 0.73 & 0 & 3 & 0.92 & -0.22 & $\begin{array}{l}\text { GQ2: I do not see a lot to be } \\
\text { thankful for }\end{array}$ & 5.75 & 1.55 & 1 & 7 & -1.40 & 1.13 \\
\hline GAD5: Restlessness & 0.60 & 0.42 & 0 & 3 & 1.47 & 1.28 & $\begin{array}{l}\text { GQ3: I am grateful to different } \\
\text { people }\end{array}$ & 6.04 & 1.26 & 1 & 7 & -1.95 & 4.05 \\
\hline GAD6: Irritability & 1.08 & 0.98 & 0 & 3 & 0.67 & -0.82 & GQ4: I don't feel grateful often & 5.08 & 1.78 & 1 & 7 & -0.71 & -0.74 \\
\hline GAD7: Feeling afraid & 1.16 & 1.07 & 0 & 3 & 0.57 & -0.92 & GQ5: I appreciate things in the past & 5.78 & 1.58 & 1 & 7 & -1.65 & 1.99 \\
\hline $\begin{array}{l}\text { O1: Optimistic about } \\
\text { future }\end{array}$ & 3.68 & 1.19 & 1 & 5 & -0.44 & -0.97 & $\begin{array}{l}\text { GQ6: I can write down a long list of } \\
\text { things to be grateful for }\end{array}$ & 5.98 & 1.38 & 1 & 7 & -1.82 & 3.09 \\
\hline O2: Expect the best & 3.14 & 1.22 & 1 & 5 & 0.15 & -1.11 & PCS1: I can get good grades if I try & 2.84 & 0.45 & 0 & 3 & -3.37 & 13.21 \\
\hline $\begin{array}{l}\text { O3: Good things will } \\
\text { happen to me }\end{array}$ & 3.63 & 1.19 & 1 & 5 & -0.40 & -0.95 & $\begin{array}{l}\text { PCS2: I can do well on tests if I } \\
\text { study }\end{array}$ & 2.80 & 0.50 & 0 & 3 & -3.10 & 11.13 \\
\hline $\begin{array}{l}\text { O4: Things will work } \\
\text { out no matter what }\end{array}$ & 3.69 & 1.24 & 1 & 5 & -0.49 & -0.97 & $\begin{array}{l}\text { PCS3: I can get good marks on } \\
\text { homework if I work at it }\end{array}$ & 2.76 & 0.56 & 0 & 3 & -2.85 & 8.95 \\
\hline H1: I feel happy & 3.28 & 1.16 & 1 & 5 & 0.13 & -1.15 & $\begin{array}{l}\text { PCS4: I cannot succeed at school } \\
\text { no matter how I try }\end{array}$ & 2.70 & 0.71 & 0 & 3 & -2.62 & 6.18 \\
\hline $\begin{array}{l}\text { H2: I have a lot of } \\
\text { fun }\end{array}$ & 2.87 & 1.15 & 1 & 5 & 0.45 & -0.74 & $\begin{array}{l}\text { PCS5: I cannot get good grades no } \\
\text { matter how I try }\end{array}$ & 2.69 & 0.69 & 0 & 3 & -2.51 & 5.70 \\
\hline H3: I love life & 3.66 & 1.27 & 1 & 5 & -0.45 & -1.06 & $\begin{array}{l}\text { PCS6: I cannot do well at tests no } \\
\text { matter how hard I try }\end{array}$ & 2.69 & 0.66 & 0 & 3 & -2.35 & 5.01 \\
\hline $\begin{array}{l}\text { H4: I am a joyful } \\
\text { person }\end{array}$ & 3.48 & 1.20 & 1 & 5 & -0.17 & -1.15 & $\begin{array}{l}\text { PCS7: I cannot get good marks for } \\
\text { my homework even if I work hard } \\
\text { at it }\end{array}$ & 2.68 & 0.68 & 0 & 3 & -2.45 & 5.61 \\
\hline $\begin{array}{l}\text { MSSS1: Special } \\
\text { person around for } \\
\text { me }\end{array}$ & 5.24 & 1.63 & 1 & 7 & -0.92 & 0.17 & PCS8: I can succeed in school if I try & 2.76 & 0.66 & 0 & 3 & -3.16 & 9.56 \\
\hline $\begin{array}{l}\text { MSSS2: Special } \\
\text { person to share joy/ } \\
\text { sorrows }\end{array}$ & 5.23 & 1.73 & 1 & 7 & -0.98 & 0.04 & & & & & & & \\
\hline
\end{tabular}

PHQ-8 Patient Health Questionnaire, GAD-7 Generalized Anxiety Disorder Screener, EPOCH EPOCH Measure of Adolescent of Well-Being, MSSS Multidimensional Scale of Perceived Social Support, PCS Perceived Control Scale, GQ Gratitude Questionnaire 
Table 3 Variable Pairs with < 20\% Different Correlations

\begin{tabular}{|c|c|c|}
\hline Variable 1 & Variable 2 & $\begin{array}{l}\text { Different } \\
\text { Correlations (\%) }\end{array}$ \\
\hline MSSS6: My friends really try to help me & MSSS7: I can count on my friends when things go wrong & 8.51 \\
\hline GAD2: Uncontrollable worry & GAD3: Too much worry & 8.51 \\
\hline $\begin{array}{l}\text { MSSS2: There is a special person with whom I can share my } \\
\text { joys and sorrows }\end{array}$ & $\begin{array}{l}\text { MSSS5: I have a special person who is a real source of } \\
\text { comfort to me }\end{array}$ & 14.89 \\
\hline PCS1: I can get good grades if I really try. & PCS2: I can do well on tests at school if I study hard. & 14.89 \\
\hline MSSS6: My friends really try to help me & $\begin{array}{l}\text { MSSS9: I have friends with whom I can share my joys and } \\
\text { sorrows }\end{array}$ & 17.02 \\
\hline $\begin{array}{l}\text { MSSS5: I have a special person who is a real source of comfort } \\
\text { to me }\end{array}$ & $\begin{array}{l}\text { MSSS10: There is a special person in my life who cares } \\
\text { about my feelings }\end{array}$ & 17.02 \\
\hline PCS4: I cannot succeed at school no matter how hard I try. & PCS5: I cannot get good grades no matter how hard I try. & 19.15 \\
\hline PCS5: I cannot get good grades no matter how hard I try. & $\begin{array}{l}\text { PCS7: I cannot get good marks for my homework, even if I } \\
\text { work hard at it. }\end{array}$ & 19.15 \\
\hline GQ1: I have a lot to be thankful for in my life & GQ6: I can write down a long list of things to be grateful for & 19.15 \\
\hline
\end{tabular}

PHQ-8 Patient Health Questionnaire, GAD-7 Generalized Anxiety Disorder Screener, MSSS Multidimensional Scale of Perceived Social Support, PCS Perceived Control Scale, GQ Gratitude Questionnaire

if I try--I can do well on tests if I study), and GQ1-6 (I have a lot to be thankful for--I can write down a long list of things to be grateful for), and we decided to keep GAD2, PCS1, and GQ1 in our network.

\section{Network estimation}

The network for all seven measures was connected, see Fig. 1. The psychometric associations, or edge-values, between 43 nodes were calculated (see Additional file 1: Appendix D in Supplementary Materials.) The mean weight of edges was 0.0177 , and 378 of the 903 edges were non-zero edges. The edges revealed positive associations within both wellness measures and mental disorder measures, but negative associations between the two groups of constructs. All within-measure items were consistently clustered together. In other words, when visualized, the networks revealed that each measure formed its own cluster. Notably, the symptoms of PHQ8 and GAD-7 had strongest inter-measure connections, even appearing to overlap and form one large cluster in the plotted network. Among the psychological wellbeing measures, the EPOCH subscales of optimism and happiness were the most highly connected, and they also had strong edges with other wellbeing scales of perceived academic control, gratitude, and social support. We found that the network with all the measures had a network density of $46 \%$, which represents the percentage of nonzero edges estimated over the total number of possible edges.

We also investigated the interconnectivity between the two constructs. The strongest negative edges across psychological wellbeing measures and symptoms of depression were PHQ2--H3 (depressed mood-I love life), PHQ2--H1 (depressed mood-I feel happy), PHQ6-PCS1 (self-blame-I can get good grades if I try),
PHQ6--MSSS8 (self-blame-I can talk to family about things), and PHQ6--H3 (self-blame-I love life.) Meanwhile, those with symptoms of anxiety were GAD6--H4 (irritability-I am a joyful person), GAD6--MSSS12 (irritability -I can talk to friends about problems), and GAD6--GQ4 (irritability-I don't feel grateful).

\section{Centrality}

The most highly central symptoms according to strength (see Fig. 2) were MSSS4 (family provides emotional help and support), PHQ6 (self-blame), and PHQ2 (depressed mood). The following symptoms were the next highest centrality statistics: H1 (I feel happy), H3 (I love life), H4 (I am a joyful person), GAD2 (uncontrollable worry), GQ1 (I have a lot to be thankful for), GAD1 (nervousness), and PCS6 (I cannot do well at tests no matter how hard I try.) Thus, three of the four happiness measures were in the top ten most central symptoms. Furthermore, we determined these symptom items with significantly higher strength values by performing a difference test between their centrality statistics, using nonparametric bootstrapping with bootnet [48]. On the other end, the least central symptoms according to strength were PCS8 (I can succeed in school if I try), O2 (expect the best), and PHQ1 (little interest/pleasure.)

\section{Bridge nodes}

To dig deeper into the community clusters form and to investigate the ties between wellbeing and symptoms of depression and anxiety, we defined two communities, one for psychopathology and the other for psychological wellbeing. We were then able to investigate the bridge symptoms between these two clusters. Calculating the bridge expected influence (the bridge statistic that neutralizes positive and negative edges) and bridge strength, 

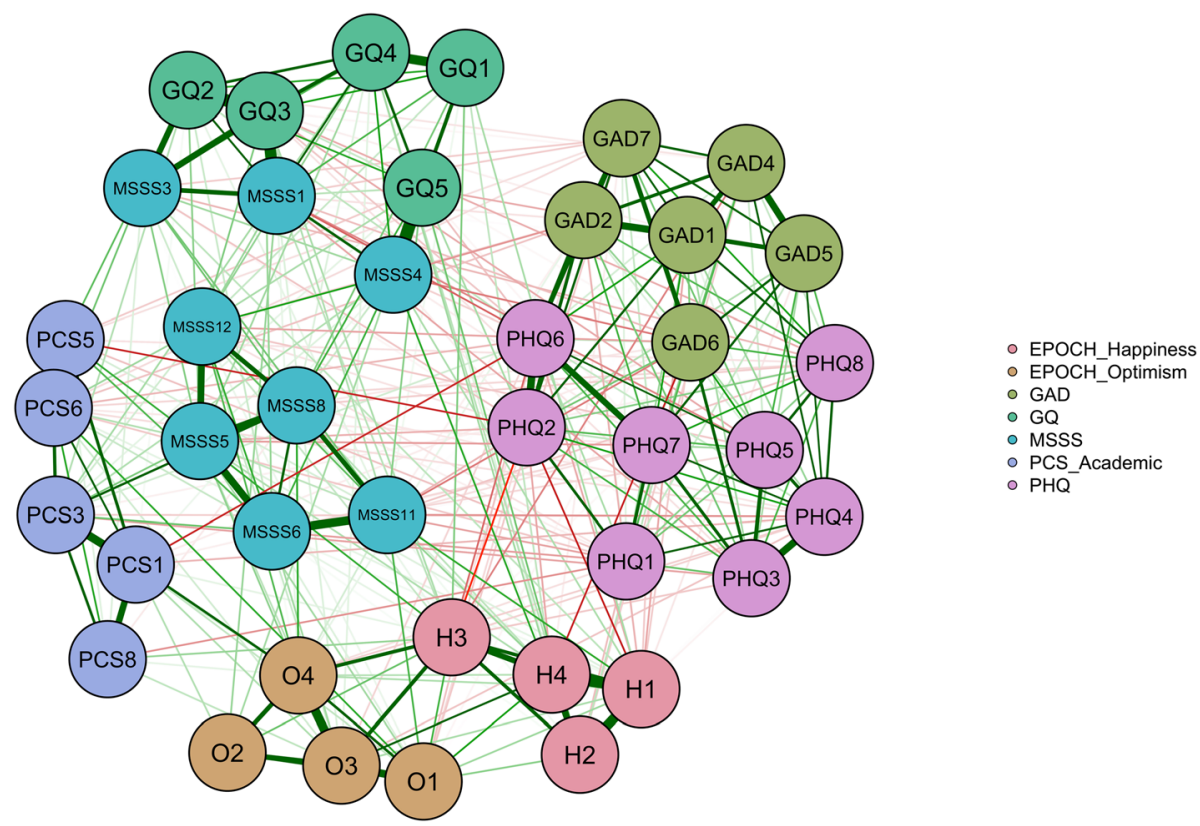

Fig. 1 Regularized partial correlation network for all measures of adolescent psychopathology and wellbeing

we identified the nodes that were most strongly and positively connected to nodes from all other measures. As can be seen in Fig. 3, the main bridge nodes were all from the Multidimensional Scale of Perceived Social Support: MSSS3 (family helps me), MSSS8 (I can talk to family about problems), MSSS11 (family willing to help me make decisions), O4 (things will work out no matter what), and MSSS12 (I can talk about my problems with friends.) The most negative bridge node was PHQ1 (little interest/pleasure), followed by PHQ2 (depressed mood) and PCS8 (I can succeed in school if I try.) Additionally, we ran bootstraps to find a good bridge strength stability with the maximum drop proportion of 0.854 to retain a correlation of 0.7 in at least $95 \%$ of the samples.

\section{Network accuracy and stability}

The bootstrapped 95\% CIs around the edge-weights were not large, meaning that the edges did not significantly vary across the bootstraps. The small CIs indicate that the edge-weights are reliable and accurate (for $\mathrm{CI}$ plots, see Additional file 1: Appendix B in Supplementary Materials.) The network also had high stability of centrality. We ran a "case dropping" bootstrap, such that we incrementally reduced the sample to determine how small we can reduce it while still maintaining a stable network. Using 15,000 bots (i.e., reduce sample, estimate network, and compute centralities fifteen thousand times), we found that $87 \%$ of the sample could be dropped before the correlation with centrality values of the full dataset dropped below $r=.7$ in at least $95 \%$ of the samples.

\section{Discussion}

We conducted a network analysis to analyze the structure of psychological wellbeing indicators and symptoms of depression and anxiety in a greatly understudied population in Sub Saharan Africa--a large community sample of Kenyan adolescents. Our results, which were robust to statistical and accuracy tests, revealed how indicators of psychological wellbeing (like happiness and gratitude) and psychopathology measures of depression and anxiety clustered in a network. We also identified the central features of adolescent psychopathology and wellbeing, as well as the interconnectedness of the various items within these domains.

This study is, to the best of our knowledge, the first attempt of its kind to combine wellbeing elements and psychopathology in a network approach with SSA youths. The recent network research of child and adolescent psychopathology has been conducted almost exclusively with Western adolescent populations $[52,57-60]$ with only a few studies in the Global South [61-63]. More research focused on SSA youths is necessary since there are scientific benefits of cross-cultural research and culture affects psychopathology and psychological wellbeing. Additionally, there is the potential utility of such research in SSA where prevalence rates for mental disorders are high [2, 21], stigma inhibits help-seeking [23], mental healthcare infrastructure is poor [2], and a large percentage of the population is youthful (the mean age in Kenya is 19.4 years [64].) Thus, our results expand our understanding of psychological wellbeing in relation to 


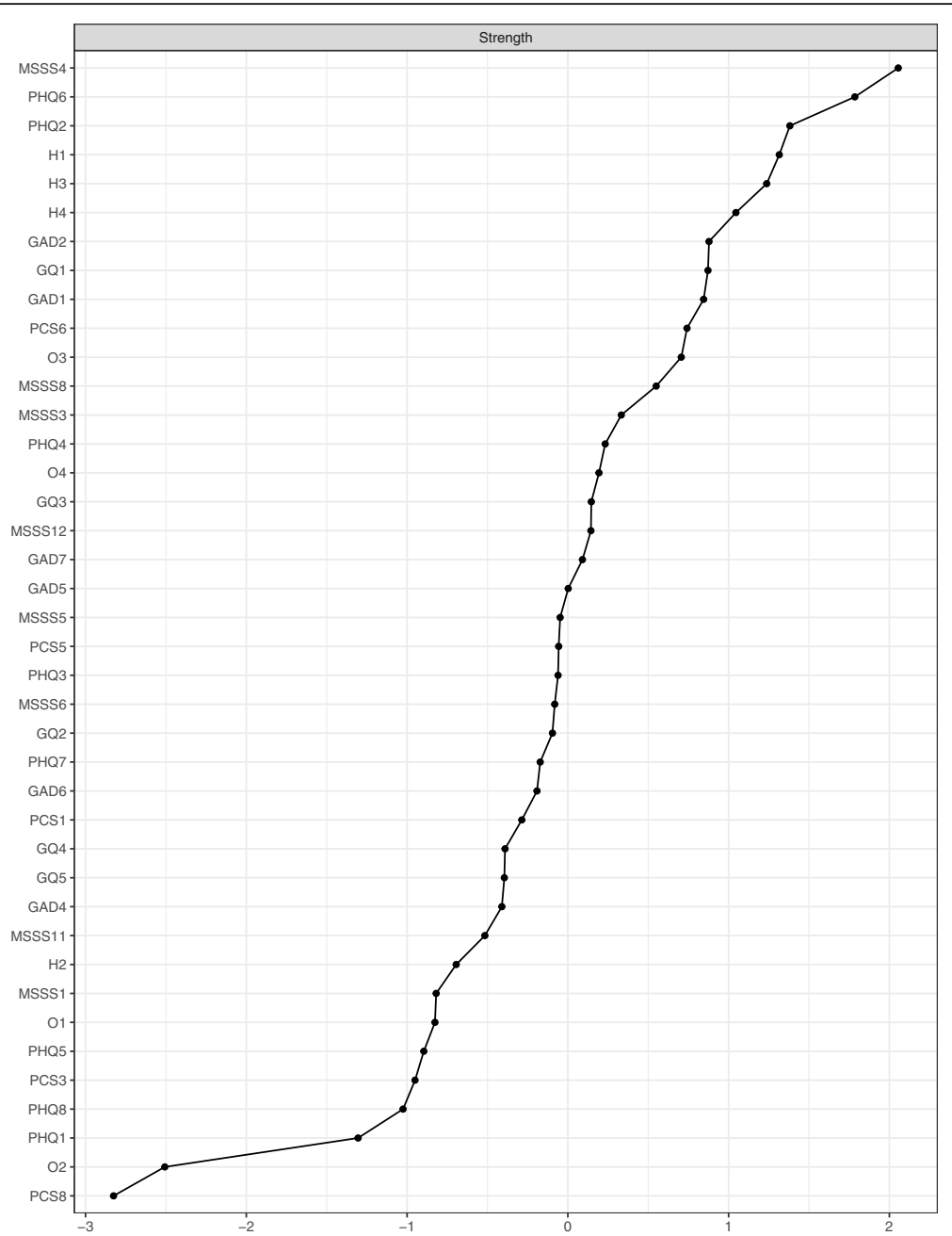

Fig. 2 Symptom centrality (strength) in a network of all measures of adolescent psychopathology and wellbeing

adolescent psychopathology in a hitherto understudied and at-risk population.

In our psychological well-being, depression, and anxiety network, two distinct clusters emerged. The constructs of psychological wellbeing (gratitude, happiness, optimism, social support, and perceived control) clustered together, while the constructs of adolescent psychopathology (depression and anxiety) formed a separate community in the network. Thus, our findings offer support for the dual factor model of psychopathology and psychological wellbeing, in which the two concepts are distinct but related constructs $[8,19]$. We found that the elements of psychopathology and those of psychological wellbeing formed two distinct clusters that were strongly and negatively associated with each other. Within the positive psychological wellbeing cluster, we found each of the constructs of psychological wellbeing to form distinct but closely connected clusters. This suggests that happiness, optimism, gratitude, and perceived control are separate but closely related constructs of psychological wellbeing. Happiness and optimism were highly connected, perhaps due to their overlapping notions or perhaps since they were subscales of the same index. Similarly, within the psychopathology cluster, anxiety and depression did not overlap but formed separate yet closely related clusters.

The wellbeing items--family provides emotional help and support, I feel happy, I love life, I am a joyful person, and I have a lot to be thankful for--as well as the psychopathology items--self-blame, depressed mood, uncontrollable worry, nervousness, and I cannot do well at tests no matter how hard I try--were the most central nodes in our network of psychological wellbeing and adolescent psychopathological, according to strength. This suggests that these items, which represent a diversity of items from different measures, may be especially important because they are strongly connected to other symptoms. Specifically, the two most central nodes in the network were family provides emotional help and support and self-blame. Emotional help and support 


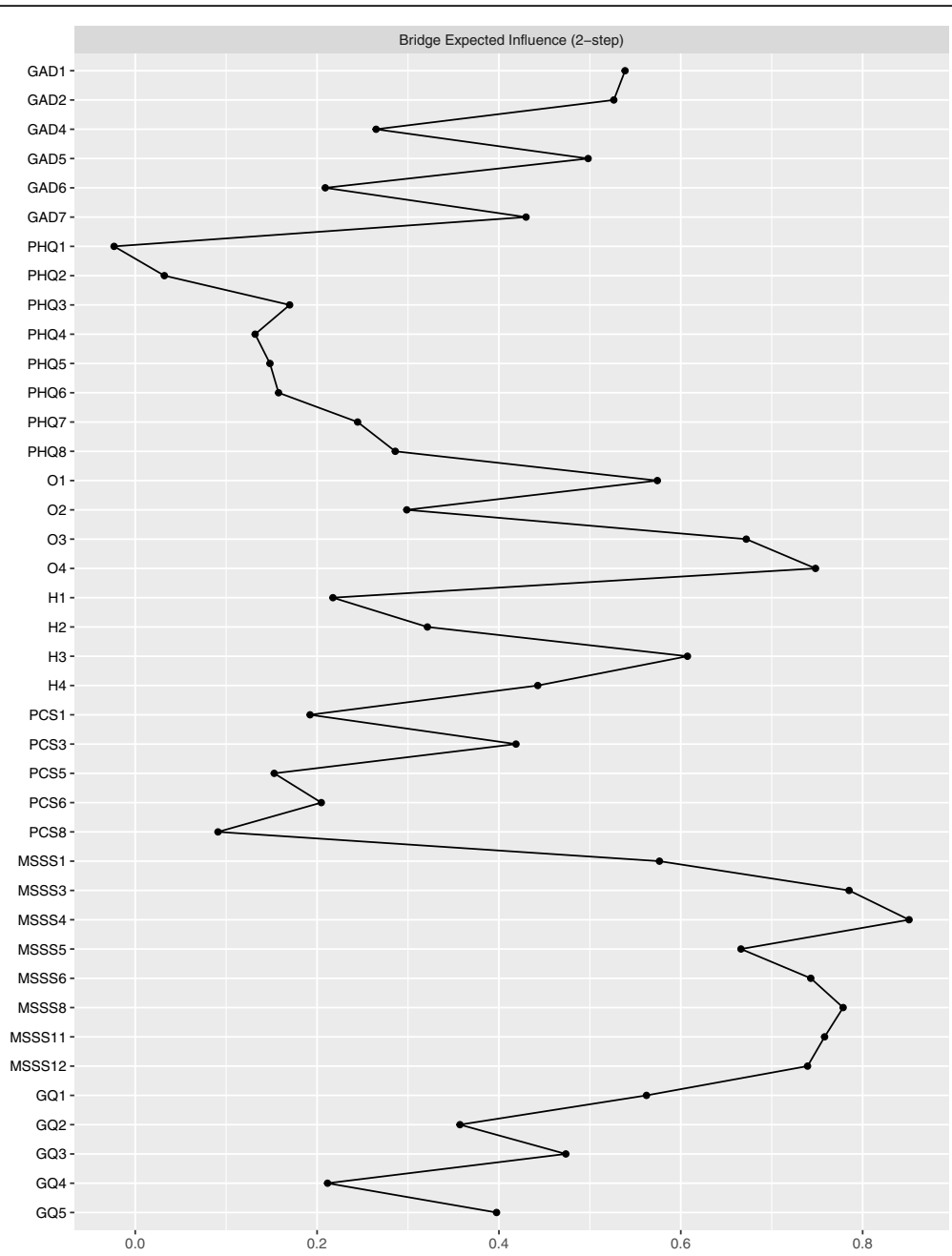

Fig. 3 Bridge items expected influence in a network with two communities for adolescent psychopathology and psychological wellbeing

from family and loved ones during this development period appear to be key to tackle negative psychopathological symptoms. As shown in Fig. 2, three of the four happiness items were in the top seven most central nodes, suggesting the potential utility of using happiness traits to uncover and prevent mental health symptoms.

Additionally, we defined two communities for the psychological wellbeing and adolescent psychopathology measures. The social support items--family helps me, I can talk to family about problems, and family willing to help me make decisions--were the most important bridge nodes that connected the two community clusters. In addition, nervousness was the most important psychopathology bridge node between the two clusters.

It is particularly striking that four of the top five strongest bridge nodes were social support items. The association between social support and mental health problems, as well as the interpretation of social support as an important protective factor against depression and anxiety, has been documented extensively elsewhere [65,
66]. In the context of Kenyan youths, it is critical to view social support in light of the nature of the Kenyan educational system. Indeed, some observers have pointed out that rather than social support, many Kenyan youths experience increased psychosocial pressure from their families, friends, and loved ones to do well in the endof-secondary school examinations [67]. As these examinations are important in determining future prospects, the external pressure to succeed from friends and families has been linked with increased depressive and anxiety symptoms amongst Kenyan youths [32]. Future studies should replicate these findings, as the association between academics, social support, and adolescent psychopathology could potentially have important public policy implications.

It is worth highlighting that little interest/pleasure emerged as the most negative bridge node. While further investigations are required to explore the means through which this symptom affects the relationship between positive wellbeing and psychopathology, one can imagine that 
having little interest/pleasure in everyday things may lead adolescents to live withdrawn lives that are absent of social support needed to improve positive wellbeing.

While these findings are insufficient to draw claims about interventions, they may suggest why positive psychological interventions that target psychological wellbeing elements rather than psychopathology-such as the Shamiri ("thrive") intervention [30] - have been successful in treating depression and anxiety symptoms with Kenyan youths. For example, research on trait gratitude suggests that having a lot of things to be grateful for is associated with exhibiting positive states and outcomes that may buffer against depression and anxiety [68]. Perhaps interventions that make salient elements of psychological wellbeing like gratitude may be effective in reducing youth depression and anxiety symptoms [31, 36, 37] because they target central elements in the network of wellbeing and psychopathology (e.g., I have a lot to be grateful for). Further studies are required to investigate this proposition, which may be particularly promising for SSA regions where social stigma around psychopathology might inhibit help-seeking.

An important strength of our study is the large sample size; however, the network connectivity that we report might not be generalized across different samples in SSA. In addition, our use of LASSO regularization to reduce false positives-which is the current "norm of practice" in many similar studies (see [52, 53], for example)-limits our ability to interpret our findings as independent of the sample. While we use this regularization technique to offer opportunities for comparison of our findings with those in the literature, it may be valuable for future research that uses network analysis to adopt regularization techniques that lend themselves to generalizability. That said, this weakness should be considered within the broader context of there being very few attempts to describe adolescent psychopathology and psychological wellbeing with a sample from SSA.

Another limitation is that we use measures that have minimal previous use with Kenyan youths. Though the psychometric properties of these studies have been studied and validated elsewhere, it is important that future studies use more culturally apt and psychometrically robust measures to replicate our findings. Finally, another limitation is that our study does not address the complicated clustering of our data or the role of sociodemographic variables in the network between adolescent psychopathology and wellbeing. We investigated the network differences and similarities between urban and rural subgroups (see Additional file 1: Appendix A in the Supplementary Materials.) Future studies are required to investigate this.

\section{Conclusions}

Our study used network analysis to investigate the relationship between youth psychopathology and psychological wellbeing in a large community sample of SSA youths. Our results suggested that adolescent psychopathology and psychological wellbeing are two distinct but related constructs. We identified important central and bridge nodes in the networks of psychopathology and psychological wellbeing. These results not only expand our understanding of the relationship between wellbeing and psychopathology in an understudied population, but they are also suggestive of future research directions that can espouse how the relationship between these two constructs can inform preventive and therapeutic efforts in low-income regions such as SSA.

\section{Abbreviations}

PHQ-8: Patient Health Questionnaire-8; GAD-7: Generalized Anxiety Disorder Screener-7; MSSS: Multidimensional Scale of Perceived Social Support; PCS: Perceived Control Scale; GQ-6: Gratitude Questionnaire - 6; EPOCH: Engagement, Perseverance, Optimism, Connectedness, and Happiness Scale; MUERC: Maseno University Ethics Review Committee

\section{Supplementary Information}

The online version contains supplementary material available at https://doi. org/10.1186/s12888-021-03331-x.

\section{Additional file 1}

\section{Acknowledgements}

We are grateful to Shamiri Institute, Inc., for granting us access to the database used for this study. We are also grateful to Benny $\mathrm{H}$. Otieno who use was helpful in data acquisition.

\section{Authors' contributions}

SC and TO conceived of the study. SC analyzed the data and drafted the methods and results section. TO acquired the data and drafted the introduction and discussion sections. SC and TO revised and reviewed the final version of the manuscript. The authors read and approved the final manuscript.

\section{Funding}

The study is funded by Shamiri Institute, Inc. based in Nairobi, Kenya.

Availability of data and materials

All data used for the present study is stored in the Open Science Framework repository and is publicly available (DOI: https://doi.org/10.17605/OSF.IO/ 8M5D9).

\section{Declarations}

Ethics approval and consent to participate

Study methods and protocols have been approved by the Maseno University Ethics Review Committee (MUERC) reference number MSU/DRPI/ MUERC/00727/19. Parental consent and written informed consent/assent were obtained for all adolescents per research ethics procedures at MUERC.

Consent for publication

Not applicable.

Competing interests

The authors declare they have no competition interests. 


\section{Received: 28 May 2020 Accepted: 18 June 2021} Published online: 03 July 2021

\section{References}

1. The Lancet. Better understanding of youth mental health. The Lancet. 2017; 389:1670. https://doi.org/10.1016/50140-6736(17)31140-6.

2. Patel V. Mental health in low- and middle-income countries. Br Med Bull. 2007;81-82(1):81-96. https://doi.org/10.1093/bmb/ldm010.

3. World Health Organization. Mental health atlas 2017. Geneva: World Health Organization; 2018.

4. Bertha EA, Balázs J. Subthreshold depression in adolescence: a systematic review. Eur Child Adolesc Psychiatry. 2013;22(10):589-603. https://doi.org/1 0.1007/s00787-013-0411-0.

5. Kessler RC, Amminger GP, Aguilar-Gaxiola S, Alonso J, Lee S, Ustün TB. Age of onset of mental disorders: a review of recent literature. Curr Opin Psychiatry. 2007;20(4):359-64. https://doi.org/10.1097/YCO.0b013e3281 6ebc8c.

6. Pine DS, Cohen P, Gurley D, Brook J, Ma Y. The risk for early-adulthood anxiety and depressive disorders in adolescents with anxiety and depressive disorders. Arch Gen Psychiatry. 1998;55(1):56-64. https://doi.org/10.1001/a rchpsyc.55.1.56.

7. Bartels M, Cacioppo JT, van Beijsterveldt TCEM, Boomsma DI. Exploring the association between well-being and psychopathology in adolescents. Behav Genet. 2013:43(3):177-90. https://doi.org/10.1007/s10519-013-9589-7.

8. Greenspoon PJ, Saklofske DH. Toward an integration of subjective wellbeing and psychopathology. Soc Indic Res. 2001;54(1):81-108. https://doi. org/10.1023/A:1007219227883.

9. Antaramian SP, Huebner ES, Hills KJ, Valois RF. A dual-factor model of mental health: toward a more comprehensive understanding of youth functioning. Am J Orthop. 2010;80(4):462-72. https://doi.org/10.1111/j.19390025.2010.01049.x

10. Zeng G, Peng K, Hu C-P. The network structure of adolescent well-being traits: results from a large-scale Chinese sample. Front Psychol. 2019;10:2783. https://doi.org/10.3389/fpsyg.2019.02783.

11. Seligman M. PERMA and the building blocks of well-being. J Posit Psychol. 2018;13(4):333-5. https://doi.org/10.1080/17439760.2018.1437466.

12. Diener E. Subjective well-being. Psychol Bull. 1984;95(3):542-75. https://doi. org/10.1037/0033-2909.95.3.542.

13. Sirgy MJ, Rahtz DR, Cicic M, Underwood R. A method for assessing residents' satisfaction with community-based services: a quality-of-life perspective. Soc Indic Res. 2000;49(3):279-316. https://doi.org/10.1023/A:1 006990718673.

14. Cohen S, Wills TA. Stress, social support, and the buffering hypothesis. Psychol Bull. 1985;98(2):310-57. https://doi.org/10.1037/0033-2909.98.2.310.

15. Watkins PC, Woodward K, Stone T, Kolts RL. Gratitude and happiness: development of a measure of gratitude, and relationships with subjective well-being. Soc Behav Personal Int J. 2003;31(5):431-51. https://doi.org/1 $0.2224 / \mathrm{sbp} .2003 .31 .5 .431$

16. Joseph S, Wood A. Assessment of positive functioning in clinical psychology: theoretical and practical issues. Clin Psychol Rev. 2010;30(7): 830-8. https://doi.org/10.1016/j.cpr.2010.01.002.

17. Winefield HR, Gill TK, Taylor AW, Pilkington RM. Psychological well-being and psychological distress: is it necessary to measure both? Psychol Well Theory Res Pract. 2012;2(1):3. https://doi.org/10.1186/2211-1522-2-3.

18. Russell JA, Carroll JM. On the bipolarity of positive and negative affect. Psychol Bull. 1999;125(1):3-30. https://doi.org/10.1037/0033-2909.125.1.3.

19. Suldo SM, Shaffer EJ. Looking beyond psychopathology: the dual-factor model of mental health in youth. Sch Psychol Rev. 2008;37(1):52-68. https:// doi.org/10.1080/02796015.2008.12087908.

20. Wilkinson RB, Walford WA. The measurement of adolescent psychological health: one or two dimensions? J Youth Adolesc. 1998;27(4):443-55. https:// doi.org/10.1023/A:1022848001938.

21. Patel V, Stein DJ. Common mental disorders in sub-Saharan Africa: The triad of depression, anxiety and somatization. In: Akyeampong E, Hill AG, Kleinman A, Akyeampong E, Hill AG, Kleinman A, editors. The culture of mental illness and psychiatric practice in Africa. Indiana University Press; 2015. p. 50-72.

22. Abbo C, Kinyanda E, Kizza RB, Levin J, Ndyanabangi S, Stein DJ. Prevalence, comorbidity and predictors of anxiety disorders in children and adolescents in rural North-Eastern Uganda. Child Adolesc Psychiatry Ment Health. 2013; 7(1):21. https://doi.org/10.1186/1753-2000-7-21.
23. Ndetei DM, Mutiso V, Maraj A, Anderson KK, Musyimi C, McKenzie K. Stigmatizing attitudes toward mental illness among primary school children in Kenya. Soc Psychiatry Psychiatr Epidemiol. 2016;51(1):73-80. https://doi. org/10.1007/s00127-015-1090-6.

24. Borsboom D. Psychometric perspectives on diagnostic systems. J Clin Psychol. 2008;64(9):1089-108. https://doi.org/10.1002/jclp.20503.

25. Fried El, van Borkulo CD, Cramer AOJ, Boschloo L, Schoevers RA, Borsboom D. Mental disorders as networks of problems: a review of recent insights. Soc Psychiatry Psychiatr Epidemiol. 2017;52(1):1-10. https://doi.org/10.1007/ s00127-016-1319-z.

26. Jablensky A. Psychiatric classifications: validity and utility. World Psychiatry. 2016;15(1):26-31. https://doi.org/10.1002/wps.20284.

27. American Psychiatric Association, editor. Diagnostic and Statistical Manual of Mental Disorders. 5th ed: American Psychiatric Association; 2013. Available: https://psychiatryonline.org/doi/book/10.1176/appi.books.9780890425596

28. Demjaha A, Morgan K, Morgan C, Landau S, Dean K, Reichenberg A, et al. Combining dimensional and categorical representation of psychosis: the way forward for DSM-V and ICD-11? Psychol Med. 2009;39(12):1943-55. https://doi.org/10.1017/S0033291709990651.

29. Borsboom D, Cramer AOJ. Network analysis: an integrative approach to the structure of psychopathology. Annu Rev Clin Psychol. 2013;9(1):91-121. https://doi.org/10.1146/annurev-clinpsy-050212-185608.

30. Osborn TL, Venturo-Conerly KE, Wasil AR, Rodriguez M, Roe E, Alemu R, et al. The Shamiri group intervention for adolescent anxiety and depression: study protocol for a randomized controlled trial of a lay-provider-delivered, school-based intervention in Kenya. Trials. 2020;21 (1):938. https://doi.org/1 0.1186/s13063-020-04732-1.

31. Osborn TL, Venturo-Conerly KE, Arango GS, Roe E, Rodriguez M, Alemu RG, et al. Effect of Shamiri Layperson-Provided Intervention vs Study Skills Control Intervention for Depression and Anxiety Symptoms in Adolescents in Kenya: a Randomized Clinical Trial. JAMA Psychiatry. 2021;1:e211129. https://doi.org/10.1001/jamapsychiatry.2021.1129.

32. Osborn TL, Venturo-Conerly KE, Wasil AR, Schleider JL, Weisz JR. Depression and anxiety symptoms, social support, and demographic factors among Kenyan high school students. J Child Fam Stud. 2020;29(5):1432-43. https:// doi.org/10.1007/s10826-019-01646-8.

33. Ndetei DM, Khasakhala L, Nyabola L, Ongecha-Owuor F, Seedat S, Mutiso V, et al. The prevalence of anxiety and depression symptoms and syndromes in Kenyan children and adolescents. J Child Adolesc Ment Health. 2008; 20(1):33-51. https://doi.org/10.2989/JCAMH.2008.20.1.6.491.

34. Kroenke K, Strine TW, Spitzer RL, Williams JBW, Berry JT, Mokdad AH. The PHQ-8 as a measure of current depression in the general population. J Affect Disord. 2009;114(1-3):163-73. https://doi.org/10.1016/j.jad.2008.06.026.

35. Spitzer RL, Kroenke K, Williams JBW, Löwe B. A brief measure for assessing generalized anxiety disorder: the GAD-7. Arch Intern Med. 2006;166(10): 1092-7. https://doi.org/10.1001/archinte.166.10.1092.

36. Osborn TL, Wasil AR, Venturo-Conerly KE, Schleider JL, Weisz JR. Group intervention for adolescent anxiety and depression: outcomes of a randomized trial with adolescents in Kenya. Behav Ther. 2020;51(4):601-15. https://doi.org/10.1016/j.beth.2019.09.005.

37. Osborn TL, Rodriguez M, Wasil AR, Venturo-Conerly KE, Gan J, Alemu RG, et al. Single-session digital intervention for adolescent depression, anxiety, and well-being: outcomes of a randomized controlled trial with Kenyan adolescents. J Consult Clin Psychol. 2020;88(7):657-68. https://doi.org/10.103 7/ccp0000505.

38. Kroenke K, Spitzer RL. The PHQ-9: a new depression diagnostic and severity measure. Psychiatr Ann. 2002;32(9):509-15. https://doi.org/10.3928/00485713-20020901-06.

39. Osborn TL, Venturo-Conerly KE, Gan J, et al. Depression and Anxiety Symptoms Amongst Kenyan Adolescents: Psychometric Properties, Prevalence, Psychosocial and Sociodemographic Factors. PsyArXiv [Preprint]. 2021 [cited 2021 Jun 27]. Available from: https://doi.org/10.31234/osf.io/ zestf.

40. Omoro SAO, Fann JR, Weymuller EA, MacHaria IM, Yueh B. Swahili translation and validation of the patient health Questionnaire-9 depression scale in the Kenyan head and neck Cancer patient population. Int J Psychiatry Med. 2006;36(3):367-81. https://doi.org/10.2190/8W7Y-0TPMJVGV-QW6M.

41. Osborn TL, Kleinman A, Weisz JR. Complementing standard Western measures of depression with locally co-developed instruments: a crosscultural study on the experience of depression among the Luo in Kenya. 
Transcult Psychiatry. 2021;1:136346152110005. https://doi.org/10.1177/13634 615211000555.

42. Kern ML, Benson L, Steinberg EA, Steinberg L. The EPOCH measure of adolescent well-being. Psychol Assess. 2016;28(5):586-97. https://doi.org/1 0.1037/pas0000201.

43. Zimet GD, Dahlem NW, Zimet SG, Farley GK. The multidimensional scale of perceived social support. J Pers Assess. 1988;52(1):30-41. https://doi.org/1 0.1207/s15327752jpa5201_2.

44. Weisz JR, Southam-Gerow MA, McCarty CA. Control-related beliefs and depressive symptoms in clinic-referred children and adolscents: developmental differences and model specificity. J Abnorm Psychol. 2001; 110(1):97-109. https://doi.org/10.1037/0021-843X.110.1.97.

45. McCullough ME, Emmons RA, Tsang J-A. The grateful disposition: a conceptual and empirical topography. J Pers Soc Psychol. 2002;82(1):112-27. https://doi.org/10.1037/0022-3514.82.1.112.

46. Wood AM, Froh JJ, Geraghty AWA. Gratitude and well-being: a review and theoretical integration. Clin Psychol Rev. 2010;30(7):890-905. https://doi. org/10.1016/j.cpr.2010.03.005.

47. Jones P. networktools: Tools for identifying important nodes in networks. R Package Version, vol. 1; 2017.

48. Epskamp S, Borsboom D, Fried El. Estimating psychological networks and their accuracy: a tutorial paper. Behav Res Methods. 2018;50(1):195-212. https://doi.org/10.3758/s13428-017-0862-1.

49. Epskamp S, Cramer AO, Waldorp L, Schmittmann VD, Borsboom D. Qgraph: network visualizations of relationships in psychometric data. J Stat Softw. 2012;48:1-18

50. Van Buuren S. Flexible imputation of missing data: CRC press; 2018. https:// doi.org/10.1201/9780429492259.

51. van Buuren S, Groothuis-Oudshoorn K. mice: Multivariate Imputation by Chained Equations in R. J Stat Softw. 2011;45:1-67. https://doi.org/10.18637/ jss.v045.i03.

52. Mullarkey MC, Marchetti I, Beevers CG. Using network analysis to identify central symptoms of adolescent depression. J Clin Child Adolesc Psychol. 2019;48(4):656-68. https://doi.org/10.1080/15374416.2018.1437735.

53. Wang SB, Jones PJ, Dreier M, Elliott H, Grilo CM. Core psychopathology of treatment-seeking patients with binge-eating disorder: a network analysis investigation. Psychol Med. 2019;49(11):1923-8. https://doi.org/10.1017/ S0033291718002702

54. Foygel R, Drton M. Extended Bayesian information criteria for Gaussian graphical models. In: Lafferty JD, Williams CKI, Shawe-Taylor J, Zemel RS, Culotta A, editors. Advances in neural information processing systems 23: Curran Associates, Inc; 2010. p. 604-12. Available: http://papers.nips.cc/pa per/4087-extended-bayesian-information-criteria-for-gaussian-graphicalmodels.pdf.

55. Robinaugh DJ, Millner AJ, McNally RJ. Identifying highly influential nodes in the complicated grief network. J Abnorm Psychol. 2016;125(6):747-57. https://doi.org/10.1037/abn0000181

56. Haslbeck JM, Waldorp L. How well do network models predict observations? On the importance of predictability in network models. Behav Res Methods. 2018;50(2):853-61. https://doi.org/10.3758/s13428-017-0910-x.

57. Jones PJ, Heeren A, McNally RJ. Commentary: a network theory of mental disorders. Front Psychol. 2017;8:1305. https://doi.org/10.3389/fpsyg.2017.013 05 .

58. Beard C, Millner AJ, Forgeard MJC, Fried El, Hsu KJ, Treadway MT, et al. Network analysis of depression and anxiety symptom relationships in a psychiatric sample. Psychol Med. 2016;46(16):3359-69. https://doi.org/10.101 7/S0033291716002300.

59. McElroy E, Fearon P, Belsky J, Fonagy P, Patalay P. Networks of depression and anxiety symptoms across development. J Am Acad Child Adolesc Psychiatry. 2018;57(12):964-73. https://doi.org/10.1016/j.jaac.2018.05.027.

60. Levinson CA, Brosof LC, Vanzhula I, Christian C, Jones P, Rodebaugh TL, et al. Social anxiety and eating disorder comorbidity and underlying vulnerabilities: using network analysis to conceptualize comorbidity. Int J Eat Disord. 2018;51(7):693-709. https://doi.org/10.1002/eat.22890.

61. Osborn TL, Campbell S, Ndetei D, Weisz JR. Network Analysis Reveals Central Symptoms of Adolescent Depression and Anxiety in Sub-Saharan Africa. PsyArXiv [Preprint]. 2020 [cited 2021 Jun 27]. Available from: https://doi. org/10.31234/osf.io/dv6c9.

62. Wasil AR, Venturo-Conerly KE, Shinde S, Patel V, Jones PJ. Applying network analysis to understand depression and substance use in Indian adolescents. J Affect Disord. 2020;265:278-86. https://doi.org/10.1016/j.jad.2020.01.025.
63. Venturo-Conerly KE, Wasil A, Osborn TL, Weisz JR, Jones PJ. Internalizing Symptoms and Social Support Among Kenyan Adolescents: A Network Analysis. PsyArXiv. 2019;1:07-22 Available: https://osf.io/f8czn.

64. United Nations D of E, Social Affairs PD. World Population Prospects 2019. 2019.

65. Cumsille PE, Epstein N. Family cohesion, family adaptability, social support, and adolescent depressive symptoms in outpatient clinic families. J Fam Psychol. 1994;8(2):202-14. https://doi.org/10.1037/0893-3200.8.2.202.

66. Wight RG, Botticello AL, Aneshensel CS. Socioeconomic context, social support, and adolescent mental health: a multilevel investigation. J Youth Adolesc. 2006;35(1):109-20. https://doi.org/10.1007/s10964-005-9009-2.

67. Yara PO, Wanjohi C. Performance determinants of Kenya certificate of secondary education (KCSE) in mathematics of secondary schools in Nyamaiya division, Kenya Asian. Sociol Sci. 2011;7(2):p107. https://doi.org/10. 5539/ass.v7n2p107.

68. Froh JJ, Kashdan TB, Ozimkowski KM, Miller N. Who benefits the most from a gratitude intervention in children and adolescents? Examining positive affect as a moderator. J Posit Psychol. 2009;4(5):408-22. https://doi.org/10.1 080/17439760902992464.

\section{Publisher's Note}

Springer Nature remains neutral with regard to jurisdictional claims in published maps and institutional affiliations.

Ready to submit your research? Choose BMC and benefit from:

- fast, convenient online submission

- thorough peer review by experienced researchers in your field

- rapid publication on acceptance

- support for research data, including large and complex data types

- gold Open Access which fosters wider collaboration and increased citations

- maximum visibility for your research: over $100 \mathrm{M}$ website views per year

At BMC, research is always in progress.

Learn more biomedcentral.com/submissions 